Revue d'histoire de l'Amérique française

ZWB REVUE D.HISTOIRE DE L'AMÉRIQUE FRANÇAISE

\title{
Réexamen de l'École des arts et métiers de Saint-Joachim
}

\section{Peter N. Moogk}

Volume 29, numéro 1, juin 1975

URI : https://id.erudit.org/iderudit/303415ar

DOI : https://doi.org/10.7202/303415ar

Aller au sommaire du numéro

Éditeur(s)

Institut d'histoire de l'Amérique française

ISSN

0035-2357 (imprimé)

1492-1383 (numérique)

Découvrir la revue

Citer cet article

Moogk, P. N. (1975). Réexamen de l’École des arts et métiers de Saint-Joachim. Revue d'histoire de l'Amérique française, 29(1), 3-29.

https://doi.org/10.7202/303415ar d'utilisation que vous pouvez consulter en ligne.

https://apropos.erudit.org/fr/usagers/politique-dutilisation/ 


\title{
RÉEXAMEN DE L'ÉCOLE DES ARTS ET MÉTIERS DE SAINT-JOACHIM *
}

\author{
Peter N. Moogk \\ Département d'histoire \\ University of British Columbia
}

L'historien se doit de retourner sans cesse aux sources pour vérifier les assertions de ses prédécesseurs. Cette attitude d'esprit se justifie non seulement par les besoins d'une méthodologie qui évolue, mais aussi par les perspectives nouvelles et les intérêts différents que l'étude du passé suscite chez les nouvelles générations de chercheurs. Un réexamen périodique des documents révèle parfois des aspects négligés d'un événement et peut mettre en lumière le sens nouveau de certains détails tenus dans l'ombre. Etant donné que toute interprétation historique passe à travers le prisme des intérêts propres de l'historien et de l'influence qu'exerce sur lui son milieu, il n'existe en réalité aucune explication définitive qui rendrait inutile une réévaluation ultérieure. C'est pourquoi il serait de la dernière imprudence pour un historien de prendre pour un fait acquis l'interprétation de ses devanciers sans en réexaminer les fondements documentaires. Le retour aux sources entraîne parfois la nécessité de corriger une interprétation généralement reçue comme évidence historique.

On peut trouver un exemple de ce rajustement dans l'histoire de la fondation par $\mathrm{Mgr}$ de Laval d'un établissement à SaintJoachim, près du Cap Tourmente. On a généralement admis depuis longtemps que le but de cette institution dont on impute la création à l'évêque, était l'apprentissage des arts et métiers, ce qui à l'époque en faisait une création assez exceptionnelle, compte tenu qu'en France, au même moment, l'apprentissage se faisait dans la boutique de l'artisan, l'atelier de l'artiste ou les manufactures royales. La fondation d'une pareille école constitue-

* Remerciements - Ce travail de revision des faits concernant l'école des arts et métiers de Saint-Joachim a été encouragé par Doreen Walker du département des Beaux-Arts de l'Université de Colombie-Britannique, qui partageait mes doutes. Les conseils judicieux de Roderick J. Barman, W. J. Eccles et Leslie F. S. Upton m'ont aidé dans cette recherche qui s'est poursuivie pendant plusieurs années. Karen Chalmers a rassemblé des extraits d'ouvrages traitant de cette institution.

\section{[3]}

RHAF, vol. 29, no 1 (juin 1975) 
rait donc un événement à la fois important et inusité en NouvelleFrance, et le fait mérite qu'on l'examine avec attention.

On connaît les détails de l'histoire tels que répétés par les historiens: vers 1668, une école d'arts et métiers existait à SaintJoachim, à quelque trente milles en aval de Québec. On en attribue la fondation à Mgr de Laval qui, de 1675 à 1690, y aurait engagé comme professeurs un certain nombre d'artistes et d'artisans. On y aurait trouvé deux sculpteurs, Samuel Genner et Michel Fauchois, et un peintre nommé Cardenas, puis, par la suite, un maçon, Claude Baillif, et deux autres sculpteurs, Denis Mallet et Jacques LeBlond de Latour. Celui-ci fut le principal animateur de l'institution qui, de 1692 à 1701, connut son apogée sous sa direction. On lui doit, ainsi qu'à ses élèves, la décoration intérieure de la chapelle du Séminaire de Québec et celle de trois églises de la côte de Beaupré. L'école préparait surtout à la décoration des églises. Mais on y apprenait aussi le travail des métaux et le tissage. L'institution disparut après 1715 et on croit que son déclin coïncide avec la mort de son fondateur en 1708. Ce dernier événement, conjugué avec la difficulté de trouver des professeurs et l'emplacement discutable de l'école, aurait été la cause de sa disparition. Malgré tout, elle exerça une influence durable sur les arts plastiques au Canada français ${ }^{1}$. Une variation récente de l'histoire fait de l'intendant Talon un cofondateur de l'institution ${ }^{2}$ et relie celle-ci à une autre école des arts et métiers située à

1 Principaux travaux donnant un compte rendu détaillé de l'école: Louis-Philippe Audet, Histoire de l'enseignement au Québec, 1608-1971, 2 vol. (Montréal, 1971), I: 160-165. - Charles-Marius Barbeau, Au Cour de Québec (Montréal, 1934), 92-112. - Detroit Institute of Arts, The Arts of French-Canada, 1613-1870 (Detroit, 1946), 8, 16-17. - Amédée Gosselin, L'Instruction au Canada sous le régime français, 1635-1760 (Québec 1911), 346-364. - J. Russell Harper, Painting in Canada (Toronto, 1966), 11-14. - Robert H. Hubbard, The Development of Canadian Art (Ottawa, 1959), 33, 38. - Graham McInnes, $A$ Short History of Canadian Art (Toronto, 1939), 14. - Gérard Morisset, Coup d'Oeil sur les Arts en Nouvelle-France (Québec, 1941), 25-27, 52; id., La Peinture traditionnelle au Canada français (Ottawa, 1960), 27-30. - Jean Palardy, The Early Furniture of French-Canada (Toronto, 1963), 376-377. - Camille Roy, Monseigneur de Laval, 1623-1708 (Québec, 1923), 62, 64-65.

2 Gérard Morisset, dans Coup d'Oeil..., est le premier à donner à Talon le rôle de cofondateur. Cette idée est développée dans son article intitulé "L'Ecole des Arts et Métiers de Saint-Joachim", paru dans les Mémoires de la Société généalogique canadienne-française, XVI, no 2 (avril-juin 1965) : 67-73. Il ne donne pas de références, mais s'est peut-être inspiré de la lettre du 21 mai 1665 citée dans ce travail ou de la lettre subséquente dont il est fait mention dans la note 42 . 
Québec ${ }^{3}$. La plupart des détails que nous venons de mentionner sont généralement admis aujourd'hui comme faits historiques.

Un regard sur l'historiographie de la Nouvelle-France nous montre que l'histoire de l'existence et de la nature de l'école de Saint-Joachim s'est lentement forgée au cours des siècles passés. On en trouve la première description dans les Mémoires sur la vie de $M$. de Laval publiés en 1761 par Louis Bertrand de Latour. Cependant, l'auteur ne parle pas d'une école des arts et métiers comme tel et ne donne aucune date de fondation. Il écrit:

\begin{abstract}
M. de Laval établit un troisième Séminaire à la côte de Beaupré dans une maison de campagne. C'étoit la plûpart des enfans de paysans, qu'on élevoit \& entretenoit grossierement \& à moins de frais. On leur apprenoit même des métiers, \& quand on en trouvoit qui avoient de l'esprit, on les faisoit passer au Séminaire de la ville [de Québec]. C'étoit une pepinière de bons ouvriers fort attachés à la maison, d'où l'on tiroit des domestiques, des fermiers, des habitans, qu'on dispersoit dans les terres du Séminaire. ${ }^{4}$
\end{abstract}

En 1663, Mgr de Laval fondait un séminaire à Québec pour former les jeunes gens à la prêtrise. En 1668, il y rattachait une école pour garçons connue sous le nom de "Petit Séminaire". Il s'agissait en réalité de deux éléments d'une même institution appelée "le Séminaire de Québec". La première fondation était davantage un lieu de résidence qu'un séminaire au vrai sens du terme, puisque les aspirants à la prêtrise faisaient leurs études au Collège des Jésuites situé à quelques pas de là. Le "Petit Séminaire" était, par ailleurs, une école élémentaire où l'on pouvait trouver de futurs candidats à la prêtrise. L'usage équivoque du mot "séminaire" rendit possible son extension à l'école établie sur les terres du Séminaire de Québec, dans la seigneurie de Beaupré.

Un article anonyme intitulé "Notice historique sur la paroisse de St. Joachim" met le lecteur sur la piste d'une date ap-

${ }^{3}$ L'idée qu'il y eut une seconde école d'arts et métiers se trouve en germe dans la description faite par Amédée Gosselin des arts décoratifs enseignés aux séminaristes de Québec, dans L'Instruction au Canada, 350. Dans Une Maîtrise d'Art en Canada, 1800-1823, p. 11, Emile Vaillancourt énonce cette idée comme une certitude. Louis-Philippe Audet, dans Histoire de l'enseignement, I : 165-166, accepte cette idée en reconnaissant que "Cette école des arts et métiers de Québec ne fut, en réalité, qu'une seule et même école avec celle de Saint-Joachim." A la fin de cette présente analyse, on jugera du postulat sous-tendant cette idée selon laquelle tout enseignement suppose l'école.

${ }^{4}$ Louis Bertrand de Latour, Mémoires sur la Vie de M. de Laval (Cologne, 1761), 99. 
proximative de fondation de l'école. L'article a été publié en 1849 dans L'Abeille, journal étudiant du Petit Séminaire de Québec. Parce qu'il était signé d'un "T", on a attribué cet article au futur cardinal Elzéar-Alexandre Taschereau. Pas plus que Latour, l'auteur ne parle ici de l'établissement d'une école d'arts et métiers par Mgr de Laval. Mais il écrit au sujet de l'évêque:

Dans le même temps qu'il ouvrait à Québec le Petit-Séminaire [...], il fondait au pied du Cap-Tourmente une espèce de ferme-modèle, où les jeunes gens qui paraissaient moins propres aux études classiques, apprenaient à lire, à écrire et à chiffrer, tout en s'appliquant aux travaux de la terre et à différents métiers ${ }^{5}$.

L'auteur ne fournit aucune preuve qui permettrait d'établir que la fondation de l'école de Saint-Joachim coïncide avec celle du Petit Séminaire, c'est-à-dire en 1668, ou aux environs de cette date. De plus, il n'utilise pas le terme "séminaire" mais parle plutôt "d'une espèce de ferme-modèle", ce qui est conforme à la vision de sa génération. Dans les années 1840, en effet, après une longue crise agricole, on tenta d'améliorer les techniques d'exploitation du sol dans le Bas-Canada, et la ferme-modèle devint un concept qui ne nécessitait aucune explication.

Quant à l'expression "l'école des arts et métiers de SaintJoachim", elle semble être le résultat d'une tentative de faire comprendre, en termes courants, l'activité polyvalente de l'institution aux lecteurs modernes. Cette expression qui n'existe dans aucun document contemporain, apparaît pour la première fois dans l'Histoire de la Colonie française en Canada de l'abbé Faillon (1865-1866). L'institution de Saint-Joachim y est présentée non comme "une espèce de ferme-modèle", mais comme "une sorte d'école d'arts et métiers" " ${ }^{~}$. En 1872, le gouvernement du Québec donnait son approbation à l'établissement d" "écoles d'arts et métiers à tendance artistique" " saient fournir la meilleure analogie avec l'école de Saint-Joachim, quoique, à ce dernier endroit, les métiers manuels ne constituaient ni les seules, ni les principales occupations. Pour P.-J.-O. Chauveau, un pionnier de l'histoire de l'éducation au Canada, l'école de Saint-Joachim correspondait, en effet, à plusieurs institutions modernes. C'était, écrivait-il en 1876, une “école, où l'on ensei-

\footnotetext{
5 Anonyme, "Notice historique sur la paroisse de St. Joachim, dans la côte de Beaupré", L'Abeille, I, no 41 (9 juillet 1849).

6 E.-M. Faillon, Histoire de la Colonie française en Canada, 3 vol. (Montréal, 1865-66), III : 263.

7 L.-P. Audet, Histoire de l'enseignement, II : 294.
} 
gnait les arts et les beaux-arts, et où il [Laval] voulait en outre former des instituteurs. Dans sa pensée, ce devait être à la fois "une école normale, une école d'agriculture et une école des arts". 8

Il existe deux tendances plus récentes dans l'historiographie de l'école de Saint-Joachim qui nécessitent des explications. Les historiens qui suivirent Chauveau firent de l'enseignement des métiers l'activité prédominante de l'école. Ils passaient ainsi de l'analogie avec les institutions des arts et métiers à l'affirmation pure et simple qu'une pareille institution existait vraiment à Saint-Joachim. Cette nuance importante dans la ligne historiographique n'était pas le résultat de preuves nouvelles, car il semble qu'avant 1910 la source principale de la plupart des auteurs restait l'article de L'Abeille.

Pour les biographes de Mgr de Laval, la fondation de l'école des arts et métiers démontrait à la fois sa piété et son sens pratique. Auguste-Honoré Gosselin y voit la preuve que l'évêque, dans sa générosité, prenait à cœur les besoins en éducation des membres les plus démunis de la société. Dans Le Vénérable François de Montmorency Laval (1901), Gosselin écrit:

Il y a [en Nouvelle-France] une classe de citoyens qui parait négligée: ce sont les hommes de métiers et les agriculteurs. Mgr de Laval pense à eux, et il fonde à Saint-Joachim cette célèbre (sic) école des arts et métiers, à laquelle est annexée une ferme modèle et même une espèce d'école normale. ${ }^{9}$

Il est peu probable que par là, Gosselin ait voulu faire de Mgr de Laval un démocrate ou un libéral. Il en faisait plutôt une caution rétrospective des idées sociales que véhiculait à l'époque l'Eglise du Québec. Ces idées privilégiaient du point de vue moral le statut de l'agriculteur et de l'artisan par rapport à celui de l'ouvrier d'usine. En dehors du clergé, par ailleurs, des hommes comme Etienne Parent et Robert-Errol Bouchette souhaitaient voir le Canada français s'engager dans les voies du commerce et de l'industrie et réclamaient un enseignement qui préparât les jeunes à ces secteurs. C'était une condamnation indirecte du système scolaire québécois, régi par l'Eglise, où les sciences et les techniques cédaient le pas aux humanités. En 1907, finalement, le gouvernement provincial accepta le principe des écoles techniques

${ }^{8}$ P.-J.-O. Chauveau, L'Instruction publique au Canada (Québec, 1876), 53.

9 A.-H. Gosselin, Le Vénérable François de Montmorency Laval (Québec, 1901), 189 . 
et durant les quinze années qui suivirent les principales villes du Québec furent dotées de ce genre d'institutions.

Le geste $d u$ gouvernement portait-il atteinte au système d'éducation contrôlé par l'Eglise? Non pas, nous dit l'abbé Camille Roy, autre biographe de Mgr de Laval. L'évêque, selon lui, avait depuis longtemps prévu les besoins en éducation du Canada français moderne, et la preuve en était l'établissement de l'école de Saint-Joachim.

Ce qu'il faut particulièrement louer en Mgr de Laval, écrit l'abbé Roy, [...] c'est le souci prévoyant avec lequel il organisa notre première école technique. Il en conçut le projet en même temps qu'il songeait à fonder le Séminaire de Québec, et c'est au pied du Cap Tourmente qu'il créa cette institution moderne. ${ }^{10}$

Non seulement l'établissement de l'école de Saint-Joachim justifiait-il la conduite de l'Eglise en matière d'éducation, mais encore il révélait chez l'évêque-fondateur cette qualité propre à tous les grands hommes: la sagesse prévoyante.

Ce n'est qu'en 1911 qu'Amédée Gosselin chercha à asseoir le récit de l'établissement de l'école de Saint-Joachim sur une base documentaire. Sa version, qui servit de guide à tous les historiens qui vinrent après lui, n'apporte comme modification substantielle que l'introduction de l'intendant Talon à titre de cofondateur de l'école. Gosselin prit au point de départ pour acquis l'existence d'une école des arts et métiers. On ne peut lui en tenir rigueur puisque tous ses contemporains tenaient cette fondation pour un fait indiscutable. Il importe cependant de garder à l'esprit que la reconstitution de Gosselin repose sur une hypothèse et non sur une certitude établie.

Bien que l'interprétation d'Amédée Gosselin présuppose comme hypothèse l'existence d'une école des arts et métiers, elle reconnaît comme incertains beaucoup d'éléments du récit traditionnel. S'il faut louer Gosselin pour son zèle et son énergie de chercheur, il faut aussi convenir que l'enthousiasme qu'il démontre pour le sujet qu'il étudie l'entraîne à cette occasion à nous présenter ce qui aurait pu se passer comme étant ce qui a dû se passer. Il n'a pas tendance à fournir des interprétations plausibles autres que celle qu'il avance. Ainsi, commence-t-il son étude de l' "Ecole

10 Camille Roy, Monseigneur de Laval, 1623-1708 (Québec, 1923), 64. Développé à partir de discours prononcés par: l'abbé Roy en 1908 et 1910 . Ce jugement a peut-être été exprimé à quelque temps seulement de la loi de 1907. 
des Arts et Métiers de Saint-Joachim" en déclarant: "l'histoire des premières années de cet établissement n'est pas beaucoup connue; les documents font défaut sur plusieurs points". Ce qui manque c'est particulièrement un document qui mentionnerait le nom du fondateur et donnerait la date de fondation. "Rien n'empêche que dès 1668 , continue pourtant Gosselin, l'évêque de Petrée ait inauguré à Saint-Joachim l'établissement en question." Une fois la tradition admise en l'absence de toute preuve, l'hypothèse devenait certitude. L'école, écrit-il encore, "montre bien le sens pratique du grand évêque". ${ }^{11}$

Toutefois, les preuves présentées par Gosselin et d'autres sources établissent clairement qu'il existait une école à SaintJoachim à la fin du XVIIe siècle. C'était une extension du Petit Séminaire de Québec où de jeunes garçons s'initiaient aux travaux manuels. A noter cependant qu'à Québec aussi les élèves du Petit Séminaire s'adonnaient à ces travaux durant leurs temps libres. Mais un certain nombre de détails restent encore à établir: par qui et à quel moment l'école fut-elle fondée? quelle était la nature de cette école et qui sont les artisans qui y furent associés? Autant de questions qui seront examinées une à une à la lumière de la preuve historique et dont les réponses seront confrontées à celles que nous a fournies le récit traditionnel.

La fondation du Petit Séminaire remonte à 1668; la date est incontestable. Peut-elle également s'appliquer à l'école de SaintJoachim? Personne n'a mentionné une date antérieure, et le recensement de 1667 fait état, pour les deux fermes du Séminaire qui s'y trouvent, des seuls tenanciers, de leurs familles et de deux serviteurs. ${ }^{12}$ La première indication selon laquelle les fermes auraient pu servir à d'autres fins que la culture nous vient d'un vieux manuscrit qui a pour titre: "Annales du Petit Séminaire de Québec". Il contient une liste des "Noms de ceux qui sont entrés au petit séminaire et qui en sont sortis". Cette liste, qui commence en 1668, mentionne pour l'automne 1676 l'admission de deux garçons qui furent par la suite envoyés au Cap Tourmente. Jusqu'en 1685, quatorze garçons furent ainsi transférés au Cap Tourmente, appellation qui désigne ici les fermes de Saint-Joachim. Quelquesuns seulement y demeurèrent jusqu'à l'âge de 18 ans; la plupart quittèrent de leur propre gré ou furent retirés par leurs parents. ${ }^{13}$

11 A. Gosselin, L'Instruction au Canada, 348.

12 Benjamin Sulte, Histoire des Canadiens-Français, 1608-1880 (8 vol., Montréal, 1882-84), IV: 74-75 (voir Romain de Trépagny et Pierre SaintDenis, tenanciers).

13 Archives du Séminaire de Québec [ASQ], Manuscrit 2, Annales du Petit Séminaire de Québec, non paginé. 
S'il y avait une école à cet endroit, elle était vraiment de petite taille. Trois des garçons mentionnés dans ces Annales font partie d'un groupe de sept jeunes gens qu'on retrouve en 1681 sur une ferme de la seigneurie de Beaupré à laquelle sont rattachées les terres de Saint-Joachim. Ils y travaillaient sous les soins de Claude Denis et d'un couple âgé. ${ }^{14}$ Par ailleurs, l'abbé Louis Soumande, qui allait devenir un bienfaiteur de l'école à la fin du siècle, résidait au Cap Tourmente en 1683. Il est douteux qu'il ait eu à l'époque le loisir d'y exercer le rôle de professeur, car il avait à desservir quatre missions..$^{15}$

Résumons la situation avant 1685. Il ne semble pas probable qu'une école ait été établie à Saint-Joachim en 1668. Tout au plus, y aurait-il eu, après 1676, sur l'une des fermes, une petite école dont, peut-être, Claude Denis fut le maître. On peut croire que les élèves aidaient aux travaux de la ferme tout en apprenant au moins le catéchisme. On ne saurait parler d'apprentissage de métiers, sauf pour un seul garçon qui fait l'objet d'une annotation des Annales en 1678: "J. B. Lamusette entré le 8 juillet fut envoyé au cap tourmente d'où il sortit ayant appris le métier de masson". Il y avait alors dans la seigneurie de Beaupré un certain nombre de maçons qui auraient pu le prendre en charge. En dehors de ces éléments, rien n'indique qu'il y ait eu un enseignement des pensionnaires, encore moins une école, à Saint-Joachim avant 1685.

La première institution de cet endroit sur laquelle nous sommes documentés s'appelle le "Petit Séminaire du Cap Tourmente". Il y fut fondé en 1685 . On retrouve son histoire dans un document manuscrit intitulé "Transcripta, 1679-1721". Ce manuscrit date de 1786 et aurait pour auteur le frère LaurentThomas Bédard. Il s'agit visiblement d'une transcription des "Annales du Petit Séminaire de Québec" dans laquelle cependant quelques détails ont été omis alors que d'autres, entièrement nouveaux, ont été ajoutés. Ces ajouts ne paraissent pas être le fait du copiste dont on trouve les commentaires en bas de pages. Il se peut qu'il ait utilisé un texte rédigé vers 1722 et contenu dans les archives du Séminaire des Missions-Etrangères à Paris. Ces archives ayant été détruites au moment de la Révolution française, il est heureux qu'une copie du texte ait pu être faite en 1786 et conservée au Canada.

14 Benjamin Sulte, Histoire des Canadiens-Français, V: 78; les jeunes gens en question sont Noël Roy, Pierre Deslauriers et Jean Lamarche ou Dumarché.

15 H. Têtu \& C.-O. Gagnon, éditeurs, Mandements, lettres pastorales et circulaires des évêques de Québec, 4 vol. (Québec, 1887-98), I: 116, "Plan général de l'état présent des missions du Canada fait en l'année 1683". 
L'histoire de l'école constitue un des nouveaux éléments que nous fournit le manuscrit Transcripta. Elle est brève et les premiers auteurs ne l'ont que paraphrasée. Elle se lit comme suit:

Etablissement du petit Séminaire du Cap tourmente. 1685.

Au mois d'octobre 1685. Mgr. de St.Vallier et Mr. le Marquis de Denonville, Gouverneur, ayant remarqué le grand bien de la bonne éducation des enfants, souhaiterent établir au Cap tourmente un petit Seminaire d'étudiants, comme celui de Québec, ce qui fut exécuté au mois de Novembre 1685. Mr. le Marquis y contribua de quatre congés, faisant la somme de 4000\# [livres]. Mr. Soumande en eut la conduite en qualité de Directeur, et Mr. Denys à qui on avoit donné la Soutanne au mois d'octobre, en qualité de préfet. ils changerent plusieurs fois de maitres d'école, ayant peine à en rencontrer un bon, ce qui fut cause que les enfants ne profiterent pas beaucoup; ils y étoient plus de 30 . en novembre 1686 . Voyant que le soutien de ce petit Seminaire étoit de grande dépense, que Mr. le Marquis ne donnoit plus de congés à cause de la guerre, et la grande difficulté d'élever des enfants d'étude à la campagne, avec des enfants de travail, on résolut de détruire entièrement le dit Seminaire, et de conserver à Québec ceux que l'on jugea plus-propres pour les études, Sçavoir le chenaye, la Durentaye et les deux labouteilleries, freres; les autres ont été renvoyés chez leurs parents.

On ne marque icy les noms de ceux qui sont venuz demeurer au petit Seminaire de Québec, et l'on ne parle point de ce qui S t rassé au Cap tourmente [référence à la liste d'élèves des Annales] 16 .

D'autres documents confirment la première partie de ce compte rendu précis. Au cours de l'hiver 1685-1686, au moment où Mgr de Saint-Vallier faisait la visite de la région, l'école était en bonne voie. "Mon principal soin dans le Cap Tourmente, écrit le visiteur, fut d'examiner l'un après l'autre trente-un enfans que deux Ecclesiastiques du Seminaire de Québec y élevoient, \& dont il y en avoit dix neuf qu'on appliquoit à l'étude, \& le reste à des mêtiers: [...] \& si on avoit des fonds pour soutenir ce petit Seminaire; on en tireroit avec le temps un bon nombre de saints Prêtres \& d'habiles artisans." 17 En juin 1687, une lettre de M. de Brisacier, envoyée de Paris au Supérieur du Séminaire de Québec, fait allusion aux difficultés de l'école: "Par vostre Seconde lettre Vous m'apprenez que ny La Mission de lIsle de Jesus,

16 ASQ, Manuscrit 6, Transcripta, 1679-1721: 53-54.

17 Jean-Baptiste La Croix de Saint-Vallier, Estat Present de l'Eglise et de la Colonie Françoise dans la Nouvelle-France (Paris, 1688), 53-54. 
ny le petit Seminaire nouvellement establi au cap de tourmente n'ont eu Les Succez avantageux dont vous me marquies les esperances et les heureux commencements par vostre premier [...] a L'esgard des jeunes enfans qui ont volonté et capacité de bien faire, J'avoue qu'il est fascheux de ne pas les ayder tous." 18

Le témoignage des Transcripta contredit l'affirmation de Louis Bertrand de Latour selon laquelle Mgr de Laval aurait fondé le "séminaire" du Cap Tourmente. On voit facilement aujourd'hui comment Latour a pu supposer que Mgr de Laval, ce grand promoteur de l'enseignement, ait fondé une école à SaintJoachim, où il passa une partie de ses dernières années. Mais la correspondance de cette époque n'appuie pas cette hypothèse. En 1685, juste avant l'établissement de l'école, l'évêque avait pensé établir une mission pour les indiens sédentaires au Cap Tourmente ${ }^{19}$, suggestion difficile à concilier avec l'idée d'une école déjà existante au même endroit. Mgr de Laval s'intéressa aux fermes de Saint-Joachim, mais, au moment de la création de l'école, l'allusion qu'il y fit, montre qu'il n'y était pas associé. Dans une lettre de février 1686 qu'il envoie de France, il blâme Mgr de Saint-Vallier pour son extravagance et sa hâte à mettre en chantier les bâtisses de l'école. "Si j'avois esté sur les lieux, dit-il, je n'aurois pu consentir." ${ }^{20}$ Voilà qui paraît bien indiquer que Saint-Vallier et non Laval doit être considéré comme le fondateur de l'école. Les réticences de Mgr de Laval ne l'empêchèrent pas de faire preuve de générosité envers les élèves de SaintJoachim, quelques années plus tard.

Les Transcripta donnent l'impression que les malheurs du "Petit Séminaire du Cap Tourmente" mirent fin aux entreprises d'enseignement à Saint-Joachim. En réalité, seuls les élèves du cours avancé (latin, philosophie, réthorique, etc.) semblent avoir été affectés. Cet enseignement fut abandonné et les quatre élèves mentionnés dans les Transcripta, tous d'origine aristocratique, retournèrent au "Petit Séminaire de Québec" où ils s'étaient inscrits en 1685. Amédée Gosselin prétend que le cours avancé a été interrompu en 1686 et que les élèves retournèrent à Québec cette année-là. ${ }^{21}$ Les documents cités plus haut indiquent cependant que le "séminaire" du Cap Tourmente fonctionnait encore 1687.

18 ASQ, Lettres $\mathrm{O}, \mathrm{N}^{\circ}$ 2, M. de Brisacier à M. de Bernières, 13 juin

19 ASQ, Lettres N, no $130: 6$.
20 ASQ, Séminaire 1, No 47, Mgr de Laval à Mgr de Saint-Vallier, 15 février 1686 .

21 A. Gosselin, L'Instruction au Canada, 358. 
en 1686; d'ailleurs, la guerre qui aurait contribué à son abandon n'a commencé qu'en 1688. Ce serait plutôt à cette date ou peu après qu'on cessa l'enseignement avancé. Quant au cours primaire, il fut maintenu. Plus tard enfin, le cours avancé devrait réapparaître.

On retrouvait deux catégories d'élèves au "Petit Séminaire du Cap Tourmente". D'une part, les élèves du cours avancé que que l'on destinait à la prêtrise et, d'autre part, ceux que les Transcripta appellent les "enfants de travail". Ceux-ci auraient été les douze enfants que, selon Saint-Vallier, l'on préparait aux travaux manuels. Ce nombre pouvait aussi comprendre quelques pensionnaires des fermes d'avant 1685 . Ces garçons, pour la plupart originaires de la campagne, restèrent à Saint-Joachim. Les Transcripta rapportent par ailleurs le transfert de jeunes du "Petit Séminaire de Québec" à celui du Cap Tourmente en 1687, 1691, 1694 et 1708. En 1699, l'intendant de la colonie notait que le Séminaire de Québec possédait "un établissement considérable au Cap-Tourmente [...] où l'on élève et instruit quantité de jeunes gens, fils d'habitants". 22

Quel enseignement dispensait l'école de Saint-Joachim après 1688 , année qui aurait été celle de la suppression du cours avancé? La première description du programme date de 1693 et rien n'indique qu'elle s'applique aux années antérieures. Elle est contenue dans une donation de Mgr de Laval, datée du 8 juin 1693. En 1680 , l'évêque fit un don au Séminaire de Québec à la condition "que ledit seminaire seroit tenu nourrir, loger, entretenir et Elever aux Estudes en Iceluy Jusqu'a la fin de la Theologie, huict pauvres Enfans de ce pays". Il se réservait le droit d'en augmenter plus tard le nombre à douze. En 1693, il modifia l'arrangement. $\mathrm{Au}$ lieu d'ajouter le nombre prévu de quatre enfants destinés à la théologie, il fut convenu que:

ledit Seminaire en prendra et recevra en sa maison et terre du Cap tourmente, ou ailleurs ou il jugera a propos Le nombre de six pauvres Enfans du dit pays de bonne mœurs et propres au travail [...] pour y estre nourris, et entretenus instruits aux bonnes mours, a la piété, a lire, et a escrire et formez au travail ou a quelqu'un des mestiers qui sy exercent Jusqu'a ce qu'ils aient atteints LAge de dix huict ans auquel ils sont en Aage de gaigner leur vie, d'Estre pris a gages et n'estre plus a charge au dit Seminaire.

22 M. de Champigny à M. de Pontchartrain, 20 octobre 1699, Rapport de l'Archiviste de la Province de Québec [RAPQ] (1939-40): 349. 
L' "ailleurs" dont il est question ici pourrait être la ferme du Séminaire sur l'Ile-Jésus, où l'on envoya un garçon en 1685. L'addition de six garçons prévus pour le Cap Tourmente plutôt que quatre destinés à Québec fut acceptée "en consideration de la plus grande charge et dépense dont ils auroient esté au dit seminaire et de l'utilité qu'il poura retirer du travail des six autres". Le Séminaire promettait aussi "de Nourrir et entretenir encor avec eux une personne capable de les Nourrir et instruire". ${ }^{23}$

Encouragé par l'exemple de Mgr de Laval, Louis Soumande fit don de 6000 livres aux mêmes conditions, le 17 juin de la même année, pour trois autres "pauvres Enfans de cedit pays". ${ }^{24}$ Il augmenta sa donation de 4000 livres en janvier 1695 pour aider le Séminaire à remplir son obligation de fournir "une personne pour conduire, Instruire aux bonnes mœurs et enseigner a lire et escrire tous les Enfans qui sont Jusques Icy fondés tant par Monseigneur de Laval [...] et par Lui Sieur Soumande qu'autres qui pourront estre fondés cy après". ${ }^{25}$ Une lettre du Séminaire des Missions-Etrangères de Paris, datée d'avril 1694, donne l'impression que l'école eut d'autres bienfaiteurs: "Vous scavez qu'on a chargé le cap Tourmente de la fondation de M. Azeur de celle du bonhomme Soumande \& qu'on a employé a cette terre de tres grosses sommes." ${ }^{26}$ On fait sans doute ici référence aux legs, en 1688, pour paiement de messes du marchand François Hazeur et du taillandier Pierre Soumande, père de Louis. Ces sommes furent offertes à Laval par le Séminaire en 1693 comme garant de sa propre dotation. ${ }^{27}$

Mgr de Laval et Louis Soumande, les deux principaux bienfaiteurs du Cap Tourmente, reçurent du Séminaire une rente viagère suffisante pour vivre. Il y eut au total neuf enfants dotés, l'abbé Soumande n'ayant pas demandé qu'on en augmenta le nombre en 1695 . Il réclama seulement, en échange de l'accroissement de sa fondation, que les enfants fassent des dévotions quotidiennes à Notre-Dame de l'Immaculée-Conception.

En octobre 1701, Louis Soumande modifia sa donation. Il fournit 8000 livres supplémentaires pour permettre "de fonder encor un maître d'Ecôle a saint Joachim, outre celui que ledit Seminaire y doit entretenir Suivant la fondation faite par

23 ASQ, Séminaire 1, No 57, Donation de Mgr. de Laval, 8 juin 1693. 24 ASQ, Séminaire 1, No 67, Donation de M. Soumande, 17 juin 1693. 1695.

25 ASQ, Séminaire 1, No 67a, Donation de M. Soumande, 20 janvier

26 ASQ, Lettres M, No 16, M. Tremblay, 6 avril 1694.

27 Voir note 23. 
Mondit seigneur Evêque, pour y instruire, conduire et enseigner tous les dits enfans fondez." Il avait, par ailleurs, reconsidéré ses dispositions à l'égard de trois protégés, et souhaitait "que les dits trois enfants seront instruits de quelques commancemens d'humanités et forméz en tout ce qui les pourra rendre propres et capables à devenir maîtres d'écoles, ou à etre employez a quelques offices dans le dit seminaire, ou dans les autres Lieux appartenans audit Seminaire". ${ }^{28}$ Onze mois plus tard, il modifia à nouveau les conditions de sa fondation, considérant "qu'il y a tres peu de prêtres en ce pais pour le deservir et la difficulté qu'il y a d'En faire venir de france". Son aide devait dorénavant permettre au Séminaire "d'En Elever deux (au lieu de trois) qui seront instruits et poussez aux Etudes Jusqu'a l'Etat ecclésiastique exclusivement". ${ }^{29}$ Ce changement avait pour effet de réduire l'aide aux enfants de Saint-Joachim, puisque les séminaristes étudiaient à Québec.

Une lettre de Louis Ango de Maizerets, supérieur du Séminaire de Québec, adressée en 1705 au Séminaire des MissionsEtrangères à Paris fait état de cette réduction: "Nous avons six enfants à Saint-Joachim suivant la fondation de Mgr. l'Ancien [Laval], qu'on appelle mitoyens parce qu'on leur apprend à lire, à écrire, et après leur étude, ils vont au travail aidant à sarcler les bleds, les jardins, à faner, à engerber les bleds." 30 On souhaitait que ces jeunes gens demeurent au service du Séminaire après l'âge de 18 ans, comme le révèle une lettre du procureur qui se plaint qu'“il n'en reste aucun à la maison, contre l'attente de Mgr. l'Ancien et de M. Soumande"..31 A Paris, le Séminaire des Missions-Etrangères s'interrogea sur la valeur de l'école et favorisa son abandon. ${ }^{32}$ Quoi qu'il en soit, lorsque Pehr Kalm visite le Cap Tourmente en 1749, il y trouve deux prêtres qui enseignent la lecture, l'écriture et le latin à des jeunes garçons dont la plupart se destinent à la prêtrise. ${ }^{33}$ Plutôt que de fermer l'école, on l'aurait donc transformée.

28 ASQ, Séminaire 1, No 68, Donation de M. Soumande, 15 octobre 1701. 1702.

29 ASQ, Séminaire 1, No 68a, Donation de M. Soumande, 27 septembre

30 ASQ, Lettres M, No 32, Louis Ango de Maizerets, 1705; citée dans Amédée Gosselin, L'Instruction au Canada, 359.

31 Voir la lettre de 1705 de M. Buisson de Saint-Cosme, citée dans Amédée Gosselin, L'Instruction au Canada, 360 .

32 ASQ, Lettres M, no 25 ( 6 juin 1698); no 29 (23 juin 1703) ; no 31 (19 juin 1705); no 67 (1729).

33 Peter Kalm (Traduit par A. Benson), Travels in North America, 2 vol. (New York, 1964), II: 481. 
Les documents établissent l'existence d'au moins deux écoles à Saint-Joachim, au pied du Cap Tourmente. D'abord le "Petit Séminaire" créé en novembre 1685 et qui dure jusqu'aux environs de 1688. Puis, l'école élémentaire, où les enfants défraient une partie de leur entretien en travaillant sur les fermes. Cette seconde école a peut-être commencé ses activités avec les quelque six enfants qu'on retrouve en pension dans les fermes après 1676 . En 1685-1686, ces "enfants de travail" qu'on utilisait dans les métiers ou sur la ferme ont doublé leur nombre. Après 1688, celuici diminue, mais sans que l'école disparaisse. De 1693 à 1702, des fonds sont prévus pour l'entretien de neuf enfants qui n'ont qu'un maître jusqu'en 1701, date à laquelle il devient financièrement possible d'en embaucher un second. En 1705, le nombre d'enfants est réduit à six. Sur la période qui va de cette dernière date jusqu'à 1749, on sait peu de choses de l'école. Les Annales et les Transcripta font mention d'un garçon qui fut envoyé de Québec au Cap Tourmente en 1708, et d'un autre de Saint-Joachim qui, sachant lire et écrire, entra au Petit Sérainaire de Québec en 1715. Le copiste des Transcripta, qui ne fait pas de distinction entre le cours élémentaire et le cours avancé, tire parti de ce dernier fait pour affirmer que le Petit Séminaire du Cap Tourmente a dû fermer ses portes en 1715 ou peu après.

Quelles preuves avons-nous de l'existence d'une troisième école à Saint-Joachim, une école des arts et métiers? On cite souvent les "Annales du Petit Séminaire" à l'appui de cette hypothèse. En plus de fournir les noms d'une vingtaine de garçons qui furent envoyés au Cap Tourmente à partir de 1676, elles donnent ceux de onze jeunes gens qui, de 1671 à 1685, apprirent les métiers de menuisier, de charpentier, de maçon, de couvreur, de cordonnier, de tailleur et de serrurier. La plupart d'entre eux avaient commencé des études scolaires et, "ne pouvant réussir", furent dirigés vers les travaux manuels. Cette excellente forme de réorientation assurait l'avenir des moins bons élèves. Le travail de ces jeunes gens comme aide aux hommes de métiers engagés a pu profiter au Séminaire, sans toutefois compenser pour les dépenses que leur entretien occasionnait et qui mettaient à rude épreuve les ressources limitées de l'établissement.

La documentation ne mentionne que pour un seul de ces apprentis le fait qu'il soit allé au Cap Tourmente. Et pourtant, depuis Faillon, tous les historiens ont confondu ce groupe avec celui des garçons qu'on envoyait à Saint-Joachim. On ne saurait certes présumer de l'identité des deux groupes qu'en se fondant sur le cas d'un seul individu. Contrairement aux élèves peu doués qui devinrent artisans, les enfants du Cap Tourmente, 
d'origine paysanne pour la plupart, y étaient envoyés sans qu'on se soit préoccupé de leurs possibilités intellectuelles. Il peut y avoir eu des critères sociaux impliqués dans la sélection. Quoi qu'il en soit, nous ne connaissons la cause que de deux transferts. En 1678, un élève fut envoyé au Cap Tourmente "ne pouvant continuer ses Etudes acause du mal de tete", ce qui tend à établir une déficience physique plutôt qu'intellectuelle.

Dans l'autre cas, l'élève ne répondait pas aux exigences spirituelles du Séminaire ou n'avait pas la vocation; on dit de lui: "après sa 1er année de théologie a été envoyé au Cap Tourmente, sous la conduite de Mr. Soumande, n'ayant pas été jugé propre pour l'état ecclesi[astique]".34 Il est peu probable que ce jeune garçon soit entré à la petite école du Cap, sauf peut-être comme instituteur. De tous ceux qui furent envoyés au Cap Tourmente, ce sont là les deux seuls cas connus d'élèves qui avaient commencé des études à Québec.

On a de bonnes raisons de croire que la majorité des élèves réorientés vers les travaux manuels restèrent à Québec. Il faut insister sur le fait que, sauf une seule exception, ils avaient fait leur apprentissage avant 1685, soit avant l'existence attestée de l'école de Saint-Joachim. Au recensement de 1681, deux d'entre eux étaient encore au Séminaire de Québec: Jacques Grouard dit LaRose et Louis Mercier, tous deux apprentis serruriers. La même année, le Séminaire avait à son service, à Québec, un boulanger, un tailleur et un serrurier. ${ }^{35}$ Aucun apprenti n'était sur les fermes du Cap Tourmente à l'époque. Tout au cours du XVIIe siècle, le Séminaire de Québec utilisa les services d'artisans français fort capables d'enseigner leur métier. Il eût été illogique, toutefois, de maintenir ce personnel coûteux à SaintJoachim, c'est-à-dire à une journée de voyage du lieu de son travail. Il y eut, bien sûr, quelques engagés sur les fermes du Cap Tourmente. Une lettre datée de 1705 y fait référence. ${ }^{36} \mathrm{La}$ plupart devaient être des ouvriers agricoles puisque la comptabilité de la "Maison de Saint-Joachim" pour la période 1702-1705 indique qu'elle dépendait des artisans de Québec pour ses confections et ses travaux spécialisés. ${ }^{37}$ Malgré tout, il dut bien y

34 Il s'agit de Robert Drouard, entré au Petit Séminaire en 1691. Voir ASQ, Manuscrit 6, Transcripta: 9.

35 Benjamin Sulte, Histoire, V : 53.

36 Voir note 31. Saint-Cosme écrit au sujet des élèves de SaintJoachim en 1705: "on tâche seulement qu'ils ne se mêlent point avec les engagés, parce que ce commerce ne leur vaut rien." Cela semble bien montrer que les engagés n'enseignaient pas aux élèves à cette époque.

37 ASQ, Manuscrit C-5, Grand Livre 1701-1723: 171-182. 
avoir quelques artisans à Saint-Joachim puisqu'on y signale la présence d'un apprenti-maçon, et qu'en 1706 le Séminaire y fit l'achat d'une forge.

A Québec, non seulement les artisans prenaient à charge les élèves peu doués, mais ils s'occupailent aussi de ceux qui poursuivaient leurs études au Petit et au Grand Séminaire. Quand Mgr de Saint-Vallier visite l'institution en 1685, il trouve les "jeunes Clercs" occupés dans leurs temps libres à de nombreux travaux manuels "non seulement pour l'utilité du domestique mais aussi pour l'ornement des Autels qu'ils parent eux-mêmes avec beaucoup de genie \& de propreté". ${ }^{38}$ Dans son Histoire de l'Amérique septentrionale (1722), Claude Le Roy de La Potherie nous donne une description du travail de ces artisans amateurs dans la chapelle du Séminaire. Par ailleurs, au XVIIIe siècle, les règlements du Petit Séminaire recommandent les travaux manuels comme façon d'utiliser les temps libres. ${ }^{39}$ L'apprentissage des arts décoratifs était à la fois utile et approprié aux séminaristes, sans compter que le patronage de Saint-Joseph ennoblissait le travail du bois. D'après Louis Bertrand de Latour, il était nécessaire pour certains séminaristes de maîtriser les travaux manuels parce qu'ils pouvaient être envoyés dans "une paroisse écartée, dépourvûe de secours". ${ }^{40}$

Même si les artisans enseignaient aux étudiants du Séminaire aussi bien qu'aux apprentis qui l'avaient quitté, ceux-ci occupaient des locaux séparés. Ils n'habitaient plus avec leurs anciens confrères. Une lettre du gouverneur Denonville au ministre des colonies en novembre 1685 décrit cette situation:

J'ay trouvé icy dans le seminaire de l'Evesché le commencement de deux establissements qui seroient admirables pour la Colonie si on les pouvoit augmenter. Ce sont Monseigneur deux maisons ou l'on retire des Enfans po[u]r les instruire dans l'une L'on y met ceux auxquels on trouve de la disposition pour les lettres, aux quels on s'atache de les former po[u]r L'Eglise [...] Dans l'autre maison on y met ceux qui ne sont propres $\mathrm{q}[\mathrm{ue}] \mathrm{po}[\mathrm{u}] \mathrm{r}$ estre artisans, et a ceux la on apprend des mestiers. Je croirois que ce seroit La un moyen admirable pour commencer un establissement de manufactures. ${ }^{41}$

38 J.-B. La Croix de Saint-Vallier, Estat Present, 13-14.

39 Amédée Gosselin, L'Instruction au Canada, 420; ce règlement est cité par L.-P. Audet, dans Histoire de l'Enseignement, I: 166.

${ }_{40}$ L.-B. de Latour, Mémoires, 99-100.

41 Denonville au Ministre, 13 novembre 1685, Archives publiques du Canada [APC] C11A, 7: 53. 
Ayant promis son soutien financier au Petit Séminaire du Cap Tourmente nouvellement établi et constaté qu'il y avait déjà des "enfants de travail" à Saint-Joachim, Denonville avait peutêtre envisagé d'y établir une manufacture. Ceci n'est que pure spéculation; par ailleurs, il serait imprudent de prétendre, comme d'autres l'ont fait par une interprétation très large des mots "dans le séminaire", que le gouverneur décrivait une école déjà existante à Saint-Joachim.

D'autres autorités de la colonie avaient déjà avancé l'idée de fabriques qui prépareraient les jeunes aux travaux manuels et allégeraient les besoins canadiens de produits importés. Avant de s'embarquer pour la Nouvelle-France en 1665, l'intendant Talon avait inclus dans ses projets pour la colonie "des atteliers pour l'utilité publique" où les artisans pourraient former des apprentis. ${ }^{42}$ C'est peut-être à partir de ce souhait qu'on a fait de Talon le cofondateur d'une école des arts et métiers. Ses efforts échouèrent, puisque son successeur, Jacques de Meulles, frappé par le coût élevé du vêtement au Canada, proposa à son tour, en 1682, que l'Etat mette sur pied une manufacture dans la colonie. De Meulles désigna l'ancienne brasserie de Québec comme emplacement possible du nouvel établissement. On y fabriquerait des vêtements et "chaque mestier prendroit icy de petits enfans, lesquels au lieu de geuser et d'estre un jour des coureurs de bois; on en feroit en les instruisant de bons ouvriers". ${ }^{33}$ La suggestion ne fut pas retenue, peut-être parce que la Métropole était réticente à rogner les fonds de ses efforts militaires en Europe pour les investir dans une aventure coloniale de faible importance. Ces projets, de même que l'idée exprimée par Denonville, tendent bien à établir qu'en France, sous l'Ancien Régime, l'apprentissage constituait une fonction secondaire de l'industrie; une école des arts et métiers consacrée principalement à l'enseignement eut été une institution vraiment unique à l'époque.

L'idée que Denonville décrivait dans son texte une école des arts et métiers située à Saint-Joachim s'appuie sur un curieux document. Il s'agit d'un manuscrit anonyme conservé au Séminaire de Québec. A l'endos, une main étrangère a inscrit la

42 Talon au Ministre, 21 mai 1665, RAPQ (1930-31): 26. Dans une lettre de novembre 1671, l'intendant déclare: “j'ay formé des asteliers qui ont entretenu près de trois cens cinquante hommes durant tout l'esté." - Ibid., 153. Sans confirmation venant d'autres sources, on ne peut identifier ces ateliers. 112-113.

43 De Meulles au Ministre, 12 novembre 1682 dans APC, C11A, 6: 
date "1685". Cependant, il semble bien que le texte et les notes soient d'époque. Amédée Gosselin attribue le manuscrit à de Meulles parce qu'il contient des vues qui coïncident avec celles de l'intendant. Si tel est le cas, on peut difficilement expliquer pourquoi ce document se trouve au Séminaire et non pas dans les Archives des Colonies à Paris. Le document concerne un projet pour l'établissement de deux manufactures au Canada. Son destinataire demeurait sans doute en France parce qu'on y parle d'envoyer des ouvriers dans la colonie, plutôt que d'en faire venir de la Métropole. L'auteur a une parfaite connaissance des travaux manuels qu'on enseignait au Séminaire de Québec. Pour cette raison, il se peut qu'il en soit un prêtre résidant, ce qui expliquerait la présence du manuscrit dans les archives du Séminaire. Comme ce document n'a été jusqu'ici cité qu'en fragments, nous voulons en reproduire des parties in extenso en mettant en italique les mots auparavant omis :

\section{Instruction Pour Establir Les Manufactures}

Pour Commencer cet ouvrage il faut de's lieux et bastiemens Commodes, il se trouve Des lieux propres pour cela, scavoir Dans La Paroisse de st.Joachim propre pour lestablissement des garçons et il sen presente un autre pour les filles dans la haute ville de Quebec, il ne reste plus qu'a faire les bastiemens et les meubles.

Le Bastiement Des garçons doit avoir Cent pieds de Long et estre a deux Estages, Lestage d'en haut pour Coucher, et LEstage d'en bas pour faire les sales des Manufactures avec deux Cheminées [...]

Les Manufactures que lon peut establir sont Des toiles Sarges, Cardeurs, fileurs de Laine, Chapeliers, Courdonniers. LOn y establira aussy Des Mestiers pour les faire apprendre aux Enfans Du Pais ce qui est desja commencé, et lon y enseigne actuellement la mesnuiserie, la sculpture, la peinture, la Dorure pour lornement Des Eglises, la Massonne, la charpente, il y a deplus tailleurs, Courdonniers, taillandiers, Serruriers, Couvreurs, qui apprennent ces Mestiers aux Enfans du Pais, mais ce qui ne peut pas se multiplier faute de logement, mais se pourra faire a la suitte a proportion Des Bastiemens que lon y fera.

Il Nest Point nécessaire denvoyer des Maistres pour enseigner les Manufactures, ny aucuns meubles ny instrumens pour lesd[its] manufactures avant que les bastiemens soi[e]nt faits, mais seullement six Massons, quattre charpentiers six mesnuisiers et tous avec leurs outils, lesquels ouvriers suffiront avec une Douzaine d'Engagés pour servir de manoeuvres tant pour le bastiement des garçons que pour celuy des filles avec les ouvriers que l'on a desja lesd[its] ouvriers doivent estre engagés pour trois ans selon lusage Du Pais. 
Le manuscrit se termine avec les caractéristiques du "bastiement Des filles", une construction de deux étages, longue de vingt pieds, qui, comme l'atelier des garçons, sera chauffée et pourra éventuellement être dotée d'ailes nouvelles. ${ }^{4}$

$\mathrm{Lu}$ dans son entier, ce manuscrit offre peu d'appui à l'idée de l'existence d'une école des arts et métiers à Saint-Joachim. Les deux manufactures sont toujours décrites au futur. On présente explicitement Saint-Joachim comme un endroit "propre pour lestablissement des garçons". Après avoir souligné les possibilités de Saint-Joachim et de la Haute-Ville de Québec comme emplacements possibles, l'auteur parle au présent de l'enseignement des travaux manuels. L'expression "on y enseigne actuellement..." est ambiguë; elle peut désigner la NouvelleFrance, ou plus spécifiquement Québec ou Saint-Joachim. On a traditionnellement admis qu'il s'agissait de Saint-Joachim. Pourtant, Québec, et en particulier le Séminaire de Québec, constituerait un choix plus logique. "La mesnuiserie, la sculpture, la peinture, La Dorure pour lornement Des Eglises" correspondent aux travaux pratiqués par les séminaristes dans leurs temps libres. Si nous reportons la menuiserie à la liste des métiers et à celle des artisans dont le texte fait état, nous constatons que l'ensemble coïncide avec les travaux manuels vers lesquels on dirigeait les élèves du Petit Séminaire incapables de suivre leurs classes. Seule la taillanderie est absente de la liste, et l'omission n'en est pas importante, car presque tous ceux qui travaillaient le métal devaient s'y initier, qu'ils fussent forgerons ou serruriers. Bref, la correspondance entre les métiers que mentionne le manuscrit et ceux qu'on enseignait au Séminaire de Québec est telle qu'il faut y voir plus que le résultat d'une simple coïncidence.

Ayant déduit l'existence d'une école des arts et métiers à partir de preuves établissant des formes d'apprentissage, des historiens comme Amédée Gosselin furent amenés à identifier les maîtres qui enseignaient à l'école. L'explication traditionnelle veut que l'école se soit consacrée particulièrement à la sculpture sur bois, et que l'enseignement de cet art ait été dispensé par Michel Fauchois, Samuel Genner, Denis Mallet et surtout Jacques LeBlond de Latour. Par ailleurs, M. Cardenas aurait enseigné la peinture et Claude Baillif, le maçonnage.

Tous ces artisans, à l'exception de Denis Mallet, furent à l'emploi du Séminaire de Québec pour une période allant de 1675

${ }^{44}$ ASQ, Polygraphie 6, no 22, Instruction Pour Establir Les Manufactures. 
à 1695. Les archives notariales indiquent que Mallet travaille pour les Récollets et les Jésuites à Québec; rien ne prouve qu'il ait été associé au Séminaire. ${ }^{45}$ Par ailleurs, les livres de compte $d u$ Séminaire montrent que Fauchois, Genner et Baillif arrivèrent à Québec en septembre 1675 venant de La Rochelle. Quant à "P.A. de Cardenas", il débarqua à Québec un an plus tard. ${ }^{46}$ Rien n'indique que ces ouvriers furent envoyés au Cap Tourmente. Apparemment engagés comme artisans, ils ont très bien pu enseigner à certains élèves du Séminaire, mais nous n'en avons pas la preuve. Pour Fauchois, nous lisons: "Michel fauchois aprentif Sculpteur Loué pour quattre ans a cent francs par an suivant lescrit de luy signé [...] Son temps commence du 21 Sept quil est arrivé a quebec." Suit une liste de créances pour biens et argent reçus terminée par la mention "Repassé en France" ${ }^{47}$. Il n'a sans doute pas terminé son engagement, puisque ses comptes ne sont inscrits que pour un an et demi. Son compagnon, Samuel Genner, n'est resté au service du Séminaire qu'un mois de plus. ${ }^{48}$ Quant à de Cardenas, les livres de comptes ne mentionnent à son sujet qu'un hiver de résidence. ${ }^{49}$ Enfin, Claude Baillif qui, selon certains historiens modernes, serait venu au Canada à l'invitation de Mgr de Laval pour enseigner à SaintJoachim, est pourtant lui-même enregistré comme engagé. Les livres indiquent qu'il "gaigne par chaque année autant que Les autre tailleurs de pierre scavoir soixante escus par an". ${ }^{50}$ Après être resté au service du Séminaire un an et quatre mois, il s'installe à son compte comme maître-bâtisseur à Québec. Donc, des six artisans dont on prétend qu'ils enseignèrent à l'école de SaintJoachim, un n'a eu aucun lien avec le Séminaire, et quatre ont été à son service moins de deux ans. Rien n'indique, par ailleurs, qu'ils soient allés à Saint-Joachim. Même s'ils ont effectivement enseigné à des élèves du Séminaire, leur trop bref séjour n'a pu avoir d'influence marquante sur les arts. Selon Amédée Gosselin: "pendant plusieurs années, Jacques Le Blond [de Latour] fut à la tête de l'atelier de sculpture, soit au Séminaire, soit à Saint-Joachim [...] il forma nombreux et habiles sculpteurs". ${ }^{11}$ Il aurait eu une influence profonde et durable sur la décoration

45 Pierre Mayrand, "Mallet, Denis", Dictionnaire biographique du Canada (1966), II: 470-471.

46 ASQ, Manuscrit C-2, Grand Livre 1674-1687: 184-185, 188-189, 224-225, 347-348, 357-358.

47 Ibid.: 185.

48 Ibid.: 189.

49 Ibid.: $357-358$.

50) Ibid.: 224-225.

51 Amédée Gosselin, L'Instruction au Canada, 361. 
religieuse au Canada français, antant en peinture qu'en sculpture sur bois. On lui doit, dit-on, à lui et à ses élèves, la décoration intérieure de la chapelle du Séminaire de Québec ainsi que le travail du bois dans les églises de Sainte-Anne de Beaupré, de l'Ange-Gardien et autres paroisses. ${ }^{52}$ Il apparaît pour la première fois, en 1695, dans les livres du Séminaire de Québec, sous le nom de "Sr. de la Tour, sculpteur, debiteur". On le désigne encore sous le nom de "Sr. Le Blond de la Tour, sculpteur", en 1696. En octobre 1695 , il reçoit ses premiers gages et son compte indique qu'en mars 1696 "Il a pris l'habit Ecclesiastique et vist en commun dans le Séminaire". ${ }^{53}$ L'auteur des Transcripta note que "Mr. Le Blond prit la Soutane le 7. Avril 1696", et le copiste de 1786 ajoute au bas de la page des commentaires qui sont à l'origine de nos connaissances sur l'influence artistique de LeBlond. Gosselin et d'autres les ont cités, en omettant toutefois les mots reproduits ici en italique:
Mr. Leblond dont nôtre Memo[ire] ne parle qu'ici pouroit être Mr. LeBlond, curé de la Baye St.Paul. Si c'est lui, on pouroit ajouter qu'il fut un excellent Sculpteur, qui forma des élèves, qui partagerent avec lui l'honneur du Sacerdoce ainsi que l'art de manier le Cizeau. Les retables de Ste. Anne, du Chateau-Richer, de l'Ange gardien, déposent en leur faveur. ${ }^{54}$

Comme dans les cas des "Instructions Pour Establir Les Manufactures", les omissions ont rendu affirmatif un texte qui ne l'était pas. Les grands livres du Séminaire parlent bien d'un sieur LeBlond de la Tour qui fut sculpteur, puis, apparemment, prêtre; il est possible qu'il ait été le curé de la Baie Saint-Paul dont parle le copiste. Mais, on a pris cette possibilité pour une certitude et on a vu dans la note plus de choses qu'elle n'en contenait. Ce qu'elle affirme c'est uniquement qu'un M. LeBlond, curé de Baie Saint-Paul, enseigna la sculpture à d'autres religieux et qu'ils travaillaient avec art, à en juger par les retables de trois églises. Nulle part, on ne fait référence à Saint-Joachim ou même à la chapelle du Séminaire. On ajoute que les élèves de LeBlond propagèrent son enseignement au point d'attirer des apprentis lä̈ques.

Le découpage de la documentation dans le sens d'une interprétation déterminée soulève la question du caractère scientifi-

52 Voir par exemple Gérard Morisset, “L'Ecole des Arts et Métiers de Saint-Joachim", dans les Mémoires de la Société généalogique canadiennefrançaise, XVI, no 2 (avril-juin 1965); l'historien de l'architecture Alan Gowans semble suivre l'approche de Morisset.

53 ASQ, Manuscrit C-4, Grand Livre 1688-1700: 474.

54 ASQ, Manuscrit 6, Transcripta: 13. 
que de l'explication traditionnelle qu'on donne à l'école des arts et métiers de Saint-Joachim. Comment Amédée Gosselin a-t-il pu construire un récit aussi vraisemblable de l'institution, à partir de la documentation dont nous venons de parler ? Sa recherche commença avec la conviction que l'école avait bel et bien existé. Comme nous l'avons vu, cette croyance paraît être le résultat des efforts du XIXe siècle pour identifier selon des concepts modernes l'enseignement disparate qui se donnait à Saint-Joachim. Forts de ce postulat, les historiens pouvaient trouver des références à l'école dans les documents les moins explicites. Quand Gosselin et plus tard L.-P. Audet font état de la phrase de Talon, d'ailleurs mal citée: "les jeunes gens se jettent dans les arts et métiers", ils se demandent avec emphase à quoi donc elle peut se référer, si ce n'est à l'école des arts et métiers de SaintJoachim ? ${ }^{55}$ Derrière cette présomption, il y a l'idée que SaintJoachim est le seul endroit en Nouvelle-France où l'on pouvait apprendre un métier. La croyance en l'existence d'une école est si forte chez Gosselin qu'elle l'amène à rejeter le témoignage des Transcripta selon lequel le "Petit Séminaire du Cap Tourmente" fut la première institution scolaire de Saint-Joachim. ${ }^{56}$ Son exposé repose en grande partie sur des preuves indirectes et jaillit de la certitude que l'école a existé. On comprend, dès lors, sa réaction impatiente devant des documents qui n'affirmaient pas sans équivoque l'existence de l'école des arts et métiers du Cap Tourmente. Il les élaguait de leurs imprécisions et de leurs ambiguïtés en sorte qu'ils puissent étayer sa propre interprétation. Il y insérait même, dans un but identique, ses propres suppositions. Ainsi, au milieu des "Instructions" il n'hésite pas à affirmer que l'auteur "propose de compléter l'établissement déjà existant à Saint-Joachim". ${ }^{57}$ Il laisse de côté des documents qui indiquent le contraire, comme le recensement de 1681, disponible à son époque sous forme d'imprimé. Il était déjà improbable que les apprentis dont les noms figurent dans les Annales aient séjourné à Saint-Joachim; identifier par surcroît les professeurs relevait de la haute fantaisie. Gosselin en puisa les noms

55 Amédée Gosselin, L'Instruction au Canada, 347; L.-P. Audet, Histoire de l'Enseignement, I: 161. Le texte de Talon est: "Les jeunes gens de Canada se desvoüent et se jettent dans les escholes pour les sciences, dans les arts, les mestiers, et sur tout dans la marine, de sorte que si cette inclination se nourit un peu, il y a lieu d'esperer que ce pays deviendra une pepiniere de navigateurs, de pescheurs, de matelots ou d'ouvriers, tous ayans naturellement de la disposition a ses ernplois." - Mémoire de Talon au Roi sur le Canada, 2 novembre 1671, RAPQ (1930-31) : 161.

56 A. Gosselin, L'Instruction au Canada, 355, note.

57 Ibid., 354. 
dans les livres du compte du Séminaire et dans sa propre imagination sans trop d'égards à la vraisemblance.

L'histoire conventionnelle de l'école des arts et métiers fut bâtie d'ingénieuse façon. Tous s'entendent pour reconnaître qu'on ne sait au juste ni quand elle fut inaugurée, ni quand elle ferma ses portes. Faute de preuves contraires, la tradition veut qu'elle ait été fondée par Mgr de Laval en 1668. L'envoi de garçons au Cap Tourmente à partir de 1676 et le fait que l'un d'eux, en 1678, y apprit le métier de maçon sont considérés comme des confirmations de la narration traditionnelle. Mais le problème, c'est précisément qu'il n'existe aucune preuve qu'il y ait eu une école à cet endroit avant la fondation du "Petit Séminaire du Cap Tourmente" en 1685 et qu'aucun document, par la suite, ne concerne directement une école des arts et métiers. Gosselin crut surmonter ces difficultés en disant: le "Petit Séminaire" de 1685 "ne devait être qu'une seule et même œuvre avec l'établissement des manufactures, et comme une extension de l'Ecole des Arts et Métiers, qui existait déjà à Saint-Joachim. Il aurait été difficile, ce semble, de faire la part exacte de l'un et l'autre." 58 Après avoir émis l'idée que le "Petit Séminaire du Cap Tourmente", qu'il définit comme un collège classique, ne peut être distingué de l'école des arts et métiers, il affirme qu'il ferme ses portes en 1686 et applique toutes les références ultérieures à la seule école des arts et métiers. Ainsi les donations de Laval et de Soumande démontreraient que l'école atteignit son apogée dans les années 1692-1701. Pourtant, l'affirmation selon laquelle l'institution disparut après 1715 remonte à la copie des Transcripta qui suggère bien que c'est le "Petit Séminaire" qui ferma ses portes cette année-là. Comme on le voit, l'histoire conventionnelle de l'école des arts et métiers est une synthèse de données relatives au collège de 1685 et à l'école élémentaire qui lui a survécu. Ce mélange de sources utilisées pouvait se justifier par l'argument selon lequel l'école des arts et métiers étant indissociable des autres, toute référence à celles-ci s'appliquait à celle-là. Le fait reste, cependant, qu'aucun document d'époque ne fait référence à une "école des arts et métiers" à Saint-Joachim.

Cependant, après étude des documents, on peut arguer qu'il reste des preuves d'un enseignement de travaux manuels à SaintJoachim et que, par conséquent, une telle école y a existé. Ce raisonnement est anachronique; il procède de la notion contemporaine qui veut que tout enseignement requiert un cadre institutionnel. Beaucoup d'historiens y ont pourtant succombé. La do-

58 Ibid., 358. 
nation que Soumande accorde en $170 \mathrm{l}$ pour permettre à trois garçons de devenir maîtres d'école a inspiré au rédacteur de L'A beille cette remarque: "voilà bien la première école normale du Canada". ${ }^{59}$ Quant à Amédée Gosselin, il a vu dans la diversité de l'enseignement donné à Saint-Joachim la preuve de l'existence de pas moins de cinq institutions: une école des arts et métiers, un collège classique, une école de latin, une école primaire et un collège d'agriculture. C'est une lettre de Maizerets datée de 1705 et décrivant le travail de six élèves sur la ferme qui fit dire à Gosselin que l'école des arts et métiers était devenue "une école d'agriculture". ${ }^{60}$

L'hypothèse selon laquelle l'école est un support indispensable à l'enseignement est admissible dans un contexte moderne, mais inappropriée pour la Nouvelle-France où la majorité des gens s'instruisaient en dehors de l'école. Dans les familles, les aînés enseignaient aux plus jeunes. Rares sont ceux qui apprirent leur métier dans des institutions; ceci est particulièrement vrai des artisans, qui, pour la plupart, se formèrent dans leur famille ou en stage d'apprentissage. Bien adapté aux conditions de la colonie, l'apprentissage privé et contracté devant un notaire était très répandu au Canada. ${ }^{61}$ Par contre, les institutions d'enseignement ne jouèrent qu'un rôle très minime dans la formation des artisans.

C'est par un examen systématique des sources notariales qu'on put découvrir l'importance de l'apprentissage en Nouvelle-France. Les historiens utilisent aujourd'hui des sources plus variées qu'à l'époque de Gosselin. Aucune de celles-ci, malgré tout, ne confirme l'existence d'une école des arts et métiers à Saint-Joachim. Les dernières recherches n'ont permis de verser au dossier qu'une seule pièce de 1701, impliquant Mgr de Laval et qui parle de "l'école de Saint-Joachim" pour garçons. ${ }^{62}$ Il s'agit vraisemblablement d'une référence à l'école élémentaire dont l'existence est bien établie pour l'époque. Ce manque de documents additionnels témoignerait en faveur du caractère défi-

59 "Notice historique" (suite), L'Abeille, I: no 42 (23 octobre 1849). $60 \mathrm{~A}$. Gosselin, L'Instruction au Canada, 359.

61 P. N. Moogk, "Apprenticeship Indentures: A Key to Artisan Life in New France", Canadian Historical Association, Historical Papers (1971): 65-83.

62 Camille Roy, Monseigneur de Laval, 65: citation de ce qui semble être une quittance notariée de Mgr de Laval à la veuve LeMercier. Je n'ai pu retrouver cet acte dans les greffes des notaires Louis Chambalon, François Genaple ou Etienne Jacob; il se trouve peut-être aux archives du Séminaire de Québec. 
nitif de l'analyse de Gosselin, s'il n'était avant tout la preuve de la faiblesse de son hypothèse. Après plus de soixante ans de recherches, les historiens seraient en droit d'attendre une preuve plus étoffée de l'existence de l'école des arts et métiers que celle qui fut fournie en 1911. On sait que Gosselin, dans ses écrits. fit aussi état de l'hôpital des frères Charron à Montréal (1692-1747) qui possédait un petit atelier où des artisans enseignaient gratuitement à des enfants pauvres et à des orphelins. Or des recherches ultérieures, dans les archives notariales, ont permis de confirmer qu'en effet des jeunes y apprirent la menuiserie, le travail des métaux et la confection des bas. ${ }^{63}$ Ainsi, le temps a-t-il donné raison à Gosselin au sujet d'une institution, mais il n'a rien fait de tel à propos de l'école des arts et métiers du Cap Tourmente. Se peut-il qu'un pareil établissement ait existé aussi longtemps sans que l'ensemble des documents que nous avons à notre disposition n'en fasse mention?

En plus de jeter le doute sur l'existence de l'école des arts et métiers de Saint-Joachim, cette analyse permet un meilleur éclairage de l'œuvre éducative du Séminaire de Québec. Créé avant tout pour recruter des jeunes et les préparer à la prêtrise, le Séminaire, par souci de charité, assuma une responsabilité beaucoup plus vaste. A partir de 1676, un certain nombre de jeunes ruraux sont mis en pension dans les fermes du Séminaire à Saint-Joachim; après 1685 , on leur fournit l'instruction élémentaire; en retour, ils contribuent aux travaux de la ferme. A Québec, les élèves qui ne satisfont pas aux rigoureuses conditions intellectuelles et spirituelles du Séminaire sont confiés aux artisans que celui-ci emploie; au moins onze garçons, et probablement davantage, font leur apprentissage de cette façon. Cet apprentissage n'avait pas besoin du cadre de l'école ; il suffisait de mettre chaque élève au service d'un artisan aui lui apprenait son métier; c'était là le mode courant d'apprentissage. Après 1675 , le Séminaire dut réduire progressivement cet enseignement étant forcé d'utiliser de plus en plus les services d'artisans canadiens indépendants, au lieu d'engagés venus de France, coûteux et, comme on l'a vu, souvent inconstants. ${ }^{64}$ Ces employés,

63 Archives judiciaires de Montréal, Greffe d'Antoine Adhémar, 16 novembre 1692; 3 février 1695; E.-Z. Massicotte. "Inventaire des documents et des imprimés concernant la communauté des Frères Charon ...", RAPQ (1923-1924) : 163-201.

${ }^{64}$ Les problèmes qu'a eus le Séminaire avec ses engagés venus de France et sa préférence croissante pour la main-d'œuvre canadienne sont étudiés plus en détails par Peter N. Moogk dans "The Craftsmen of New France" (thèse de Ph.D., Université de Toronto, 1973, non publiée), 109-112. 
attachés au Séminaire pour de longues périodes, étaient cependant les mieux placés pour initier à leurs métiers les élèves de l'institution. Les conditions de leur engagement ne leur permettaient pas de refuser des apprentis.

L'apprentissage chez des artisans employés par le Séminaire de Québec se poursuivit au XVIIIe siècle. En 1715, le tuteur d'un enfant (qui était apparemment un neveu orphelin) se plaignit à l'intendant du fait que le garçon s'était sauvé du Séminaire pour trouver asile chez d'autres parents, violant ainsi son contrat d'engagement "à M.rs du Séminaire de cette ville [Québec] pour les servir jusqu'a laage de quinze ans a condition qu'ils luy feront apprendre chez Eux le metier de Cordonnier a lire et Ecrire qu'ils seront chargés de sa nourriture et Entretien". ${ }^{65}$ Ce que l'on sait des jeunes apprentis du Séminaire et des pensionnaires de ses fermes permet de mieux apprécier l'éloge que fit le roi en 1711 du travail d'éducateur des prêtres de l'institution. ${ }^{66}$ Ceuxci reçurent une aide financière et en 1730 les autorités coloniales qui cherchaient à l'augmenter déclarèrent que le Séminaire acceptait autant d'élèves qu'il était possible d'en recevoir, même ceux qui ne pouvaient payer ni leurs cours ni leurs pensions. ${ }^{67}$ Cette générosité n'était pas limitée aux seuls séminaristes. L'institution assuma le fardeau additionnel de préparer des jeunes à des carrières laïques et ce, pendant la majeure partie du Régime français. Si l'on excepte Edward R. Adair de l'Université McGill, les historiens qui ont traité des beaux-arts, des arts appliqués et de leur enseignement au Canada n'ont jamais mis en doute l'existence de l'école des arts et métiers de SaintJoachim. En 1929, Adair émit l'avis que "there is indeed no real evidence that this school ever played a part in the development of French Canadian art". ${ }^{68}$ S'il y eut d'autres personnes de son avis, elles ne se sont pas manifestées. La plupart des historiens se sont contentés de reproduire le récit d'Amédée Gosselin, ses extraits de documents et même ses notes en bas de pages. Là où il manifestait de la prudence, ils furent téméraires; ce qu'il

65 Archives du Québec, NF 2, Ordonnances des Intendants, VI: ff.151 vo-152. Le tuteur était incidemment de Saint-Joachim, tandis que son pupille venait de Château-Richer.

${ }_{66}$ Archives Nationales de France, Archives des Colonies, Série B, 33: 77vo-78 (Mémoire du Roi à Vaudreuil, 7 juillet, 1711).

67 Ibid., Série C11A, 106: 300-302 (Beauharnois et Hocquart au Ministre, 21 décembre 1730).

68 E. R. Adair, "French Canadian Art", Canadian Historical Association, Annual Report (1929): 93. 
présentait comme possibilités devint faits irréfutables. A son récit, ils ajoutèrent leurs propres embellissements sans se poser de questions sur son fondement historique.

Le but de cet article est précisément d'attirer l'attention sur le fait que l'histoire de l'école des arts et métiers ne s'appuie sur aucune certitude. Il reste beaucoup à faire en histoire de l'enseignement et des beaux-arts en Nouvelle-France. Cet exemple montre qu'on ne peut toujours prendre pour acquis les affirmations passées. Il est prudent de commencer par un examen critique de la preuve.

Traduction de JEAN BLAIN

Université de Montréal 